\section{Cell-targeted phagemid particles preparation using Escherichia coli bearing ligand-pIII encoding helper phage genome}

\author{
Zonghai $\mathrm{Li}^{1}$, Hua Jiang ${ }^{1,2}$, Jie Zhang ${ }^{1}$, and Jianren $\mathrm{Gu}^{1,2}$ \\ 'Shanghai Jiao Tong University, Shanghai and 'Medical College of Fudan University, \\ Shanghai, China
}

BioTechniques 41:706-707 (December 2006)

doi 10.2144/000112294

The filamentous phages M13, f1, and fd have been widely applied in biological research such as phage display library, phage gene delivery, and antigen preparation (1). For phagemidmediated gene delivery, we have previously reported that modified helper phage M13KO7P1, which encodes a 12-mer ligand peptide and pIII fusion, could be used to rescue phagemid that encodes the gene of interest (2). The phagemid particles display a high level of peptide. Moreover, the phagemid is not necessary to encode the pIII protein and therefore leaves a larger space for cloning genes of interests. Since the size of phagemid particle is directly proportional to the size of the phagemid genome as previously described (3), the modified phagemid particles are expected to be smaller than those prepared using other methods. The reduced size of the gene delivery particle might enhance its penetration capacity when used in in vivo gene delivery. However, the yield of M13KO7P1 helper phage is relatively low, $3.4 \times$ $10^{9}$ plaque-forming units (pfu) $/ \mathrm{mL}$ (2). When helper phage is modified with a moderate size ligand, such as epidermal growth factor (EGF), the yield of the helper phage is even much lower, 1.5 $\times 10^{8} \mathrm{pfu} / \mathrm{mL}$ (unpublished data). To solve this problem, we made LMP cells by transforming EGF-modified helper phage genome (plasmid) into $\mathrm{F}^{+}$ bacterial cells. The phagemid encoding genes of interest was then transformed into the LMP cells to make phagemid particles.

EGF-modified helper phage genome M13EGFKO7CT: EGF encoding sequence was amplified from pAE-8 (4) using primers M13EGF-1: 5'-CGGG TACCTTTCTATTCTCACTCTA ATTCCGACTCTGAATGCCC-3' and M13EGF-2: 5'-GTTTCGGC GAACCTCCACCACGCAGTTCCC ACCATTTC-3', thus generating DNA fragment 1 . The DNA fragment 2 was amplified from M13KO7 using primers CT-1: 5'-CTGCGTGGTGG AGGTTCGGCTAGCGGTGGTGGCT CTGGTTCCGGT-3' and CT-2: 5'CGCGCAGAGGCGAATTATTC-3' . DNA fragment 3 was obtained by assembly PCR using DNA fragment 1 and 2 as template. The DNA fragment 3 encodes EGF-pIIICT (fusion protein of EGF and the carboxyl domain of protein III). DNA fragment 3 was digested with $K p n \mathrm{I}$ and $P a c \mathrm{I}$ and then ligated into M13KO7P1 (2) KpnI/PacIdigested backbone. Since the restriction
A

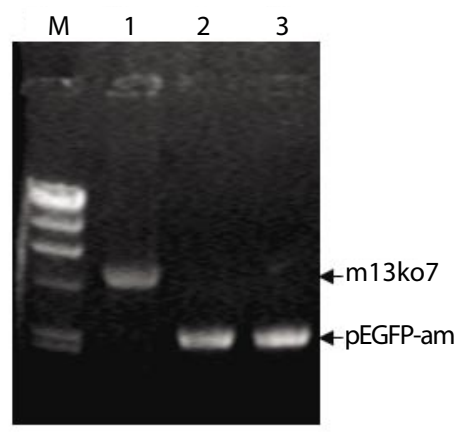
rescued by LMP cells. site of $K p n I$ was located in the leader sequence of peptide-pIII, no peptidepIII encoding sequence remained in the backbone.

The plasmid M13EGFKO7CT was transformed into $\mathrm{F}^{+}$ER2738 cells (New England BioLabs, Ipswich, MA, USA). The resulted cells were designated as LMP cells. LAM cells were stably maintained by kanamycin selection. Phagemid pEGFP-amp, which encodes enhanced green fluorescent protein (EGFP), was then transfected into LMP cells and plated in ampicillin/ kanamycin LB plates. A single colony was picked and transferred into LB medium containing the same antibiotics. The supernatant was collected and precipitated by $6 \mathrm{M}$ polyethylene glycol (PEG)/NaCl. The phages were titrated by enzyme-linked immunosorbent assay (ELISA) as previously described (5). The yield of the resulted phagemid particles reached $1.5 \times 10^{12}$ $\mathrm{pfu} / \mathrm{mL}$, about one-half of phagmid particles rescued by M13KO7 helper phage $\left(2.8 \times 10^{12} \mathrm{pfu} / \mathrm{mL}\right)$, indicating that the presented method can prepare phagemid particles efficiently.

The single-stranded DNA from the phagemid particles was prepared as before (2). As shown in Figure $1 \mathrm{~A}$, similar to the phagemid particles prepared by rescuing pEGFP-amp with M13KO7 helper phage, phagemid particles made by LMP cells mainly contained pEGFP-amp phagemid.
B

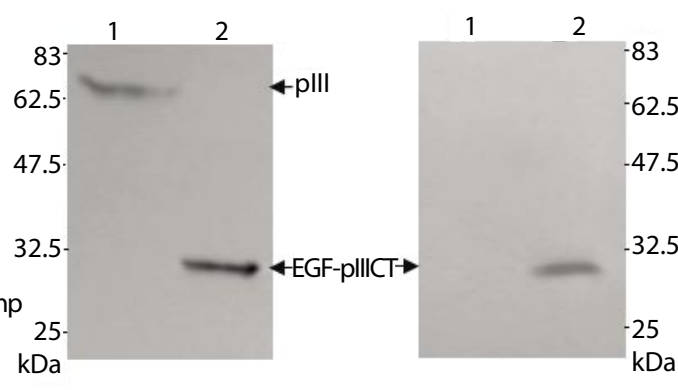

Figure 1. Analysis of phages and phagemid particles. (A) DNA released from phages and phagemid particles was loaded on a $1 \%$ agarose gel. Lane M: $\lambda$ DNA/HindIII (purchased from Takara Bio, Dalian, China); lane 1, M13KO7 helper phage; lane 2, pEGFP-amp phagemid particles rescued by M13KO7; and lane 3, pEGFP-amp phagemid particles rescued by LMP cells. (B) Western blot analysis of pIII or pIIICT in phagemid particles using anti-pIIICT antibody. Lane 1, pEGFP-amp phagemid particles rescued by M13KO7; lane 2, pEGFP-amp phagemid particles rescued by LMP cells. (C) Western blot analysis of epidermal growth factor (EGF) displayed in phagemid particles using anti-EGF antibody. Lane 1, pEGFP-amp phagemid particles rescued by M13KO7; lane 2, pEGFP-amp phagemid particles 

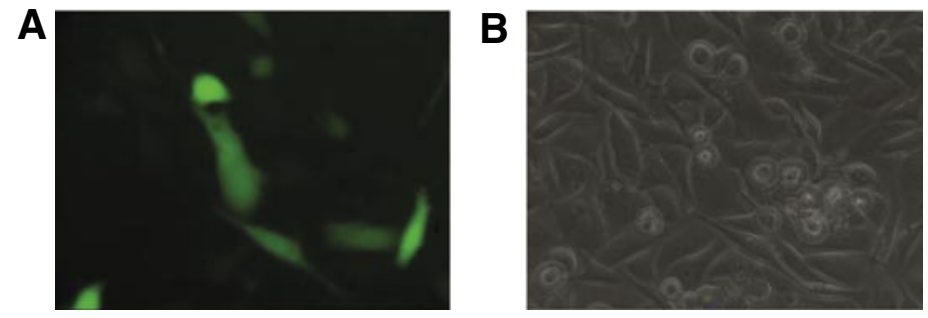

Figure 2. In vitro transfection of pEGFP-amp/LMP cells phagemid particles in PC-3 prostate cancer cell line. (A) Cells visualized under a fluorescent microscope. (B) Phase contrast image of the cells in the same field as panel A.

Almost no helper phage genome was detected. Therefore, LMP cells also can selectively package phagemids, which contains the wild-type F1 origin.

To examine the level of EGF displayed, Western blot analysis was conducted mainly following the previous protocol (2). Approximately $10^{10}$ phages were loaded per lane on a $15 \%$ polyacrylamide gel. Blocking was performed with $2 \%$ skim milk powder in phosphate-buffered saline (PBS) for $2 \mathrm{~h}$ at room temperature. Immunostaining was done with the mouse monoclonal antibody (MAb) anti-m13 pIII (New England BioLabs) recognizing the $\mathrm{C}$ terminus of pIII coat protein of $\mathrm{M} 13 \mathrm{KO} 7$ and peroxidaseconjugated goat anti-mouse immunoglobulin $\mathrm{G}(\mathrm{IgG})\left(\mathrm{H}^{+} \mathrm{L}\right)$ (KangChen, Shanghai, China), visualized with a SuperSignal ${ }^{\circledR}$ West Pico kit (Pierce Biotechnologies, Rockford, IL, USA). As shown in Figure 1B, no degraded pIIICT was detected, suggesting that all the pIIICT displayed EGF. To further demonstrate that EGF was displayed, rabbit polyclonal antibody against human EGF (Santa Cruz Biotechnology, Santa Cruz, CA, USA) and peroxidase-conjugated goat antirabbit $\operatorname{IgG}\left(\mathrm{H}^{+} \mathrm{L}\right)$ (KangChen) were used in Western blot analysis. Results shown in Figure 1C indicated that the EGF was displayed in the pIIICT. Taken together, all the pIIICT displayed EGF.

PC-3 cells (2000 cells/well) were plated in a 24-well plate for $24 \mathrm{~h}$ before transduction. Phages were then added into the medium at $10^{11}$ pfu/mL. Substantial GFP expression was observed in about $10 \%$ of the cells (Figure 2).

From data presented above, we concluded that the present method can be utilized very conveniently to prepare phagemid particles with a high level of ligand display. It is well-known that the inefficiency of replication of singlestranded genome carried by the phagebased vectors leads to low expression in mammalian cells. Therefore, some strategies have been applied to overcome this problem. One of them is using the inverted terminal repeat to enhance the replication of the singlestranded genome. AAV/phage hybrid vectors have been used in in vivo for cancer gene therapy and molecular imaging (6). Using the LMP cells, we have packaged phagemid carrying ITR of AAV efficiently. The smaller size of phagemid particles produced by this method may improve the gene transfer in vivo. However, further studies need to be performed.

\section{ACKNOWLEDGMENTS}

This work was supported by National 973 Basic Research Program Project from Science and Technology Commission of Shanghai Municipality (no. 054119568).

\section{COMPETING INTERESTS STATEMENT}

The authors declare no competing interests.

\section{REFERENCES}

1.Larocca, D. and A. Baird. 2001. Receptormediated gene transfer by phage-display vectors: applications in functional genomics and gene therapy. Drug Discov. Today 6:793-801.

2.Li, Z., J. Zhang, R. Zhao, Y. Xu, and J. Gu. 2005. Preparation of peptide-targeted (ITR) of adeno-associated virus (AAV) of China (no. 2004CB518802) and phagemid particles using protein III-modified helper phage. BioTechniques 39:493-497.

3. Mount, J.D., T.I. Samoylova, N.E. Morrison, N.R. Cox, H.J. Baker, and V.A. Petrenko. 2004. Cell targeted phagemid rescued by preselected landscape phage. Gene 341:59-65.

4. Gan, B.R., P. Huang, Y. Yuan, J. Yao, H. Wen, and Z. Wen. 1992. Secretion of overproduced human epidermal growth factor in Escherichia coli. Acta Biochim. Biophys. Sin. 24:611-615.

5. Rondot, S., J. Koch, F. Breitling, and S. Dubel. 2001. A helper phage to improve single-chain antibody presentation in phage display. Nat. Biotechnol. 19:75-78.

6.Hajitou, A. M. Trepel, C.E. Lilley, S. Soghomonyan, M.M. Alauddin, F.C. Marini 3rd, B.H. Restel, M.G. Ozawa, et al. 2006. A hybrid vector for ligand-directed tumor targeting and molecular imaging. Cell 125:385-398.

Received 23 June 2006; accepted 24 August 2006.

Address correspondence to Zonghai Li or Jianren Gu, State Key Laboratory of Oncogenes and Related Genes, Shanghai Cancer Institute, No.25/2200, Xie Tu Road, Shanghai 200032, China. e-mail: Zonghaili@gmail.com; nlorg@sh163.net

To purchase reprints of this article, contact: Reprints@BioTechniques.com 